\title{
Calcolosi cistinica: trattamento integrato e personalizzato
}

\section{A. Trinchieri}

S.C. Urologia, Ospedale A. Manzoni, Lecco

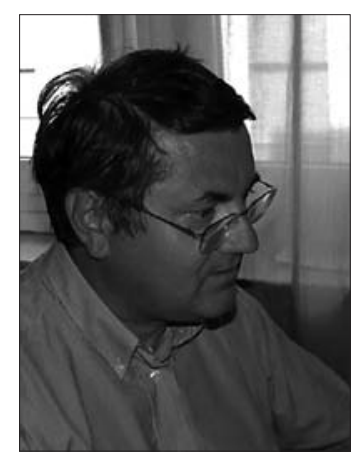

\section{Definizione}

La cistinuria è una malattia autosomica recessiva caratterizzata da un difetto del trasporto della cistina e degli altri aminoacidi dibasici (ornitina, arginina e lisina) che comporta alterazioni nel riassorbimento sia a livello del tubulo renale che del tratto gastrointestinale.

La malattia è caratterizzata da concentrazioni anormalmente elevate di cistina e degli altri aminoacidi dibasici nelle urine, con conseguente rischio di formazione di calcoli renali di cistina a causa della sua ridotta solubilità.

\section{Genetica}

La cistinuria non è causata da alterazioni di un singolo gene, in quanto sia mutazioni del gene SLC3A1, localizzato sul cromosoma $2 \mathrm{p}$ e codificante la proteina rBAT, che del gene SLC7A9, localizzato sul cromosoma 19q13.1 e codificante la proteina $\mathrm{b} 0,+\mathrm{AT}$, causano alterazioni del sistema di trasporto degli aminoacidi (complesso rBAT/ b0,+AT) (1-6). Altri geni potrebbero essere coinvolti nel processo di trasporto della cistina, dando una spiegazione genetica per quei pazienti cistinurici senza alterazioni dei due suddetti geni.

\section{Classificazione}

La cistinuria è stata classificata in tre sottotipi da Rosenberg (tipo I, II e III). La classificazione tradizionale è basata sulle caratteristiche dell'escrezione di cistina e degli aminoacidi dibasici negli eterozigoti obbligati (7).
Nella cistinuria tipo I, i parenti eterozigoti dei pazienti affetti dalla malattia hanno livelli normali di cistinuria, mentre nel tipo II gli eterozigoti hanno livelli aumentati e nel tipo III aumentati ma in minor entità rispetto al tipo II.

I progressi in campo di genetica molecolare rendono ora possibile una correlazione tra genotipo e fenotipo, per cui, conseguentemente, la cistinuria è stata classificata in tipo A, correlato alle mutazioni di SLC3A1 e caratterizzato del fenotipo I di Rosenberg e tipo B, correlato a mutazioni di SLC7A9 e corrispondente ai fenotipi non-I (II e III).

\section{Epidemiologia}

La reale incidenza del difetto metabolico nella popolazione generale è tuttora incerta. $\grave{E}$ stata stimata in un caso ogni 7000 nascite, ma i pazienti che formano calcoli di cistina rappresentano solo il 3-59\% di tutti i soggetti cistinurici (8-12).

Recentemente è stato confermato che solo il $42 \%$ dei topi SLC7A9 knockout, con elevati livelli di escrezione di cistina ed aminoacidi dibasici, sviluppa calcolosi urinaria (13).

\section{Calcolosi cistinica}

La frequenza complessiva dei calcoli di cistina tra tutti i pazienti con nefrolitiasi si aggira tra l'1 ed il $6 \%(14,15)$, tuttavia i pazienti con calcolosi cistinica rappresentano un rilevante problema clinico a causa dell'elevata frequenza di recidive con conseguente necessità di ripetuti interventi per la rimozione dei calcoli.

I calcoli di cistina possono essere "puri" o "misti" di cistina ed ossalato di calcio.e possono essere sospettati in presenza di un'evidenza radiologica di calcoli 
debolmente radiopachi.

L'esame microscopico ha consentito di caratterizzare i calcoli di cistina in base alle loro caratteristiche di superficie in "rough" e "smooth". I calcoli del primo tipo risultano molto più suscettibili alla frammentazione extracorporea rispetto a quelli del secondo tipo (16).

Kim ha potuto identificare calcoli con struttura TC più disomogenea (assimilabili al tipo "rough") che appaiono più suscettibili di quelli a struttura omogenea ("smooth") all'azione delle onde d'urto (17).

Un'analisi accurata del calcolo (spettroscopia agli infrarossi, cristallografia) può identificare con accuratezza la natura cistinica del calcolo, mentre il semplice esame chimico qualitativo del calcolo può misconoscerne la presenza quando questa rappresenti una quota minore di un calcolo misto. Analogamente l'esame chimico qualitativo può dimostrarsi poco sensibile nell'analisi di piccoli frammenti espulsi dopo litotrissia extracorporea. Talvolta la calcolosi cistinica viene diagnosticata molti anni dopo l'esordio clinico a causa della mancata identificazione all'esame del calcolo.

Conseguentemente lo screening della cistinuria dovrebbe essere eseguito in tutti i pazienti con nefrolitiasi o almeno in quelli con familiarità ed esordio prima dei 30 anni di età.

Il test di Brand (nitroprussiato di cianuro) consente di identificare la presenza di cistina nelle urine quando questa supera i $75 \mathrm{mg} / \mathrm{gm}$ di creatinina, tenuto conto che i soggetti normali eliminano con le urine meno di $100 \mathrm{mg}$ di cistina al giorno. False positività possono essere provocate dall'assunzione di medicinali (acetilcisteina ecc). La cistinuria può anche essere diagnosticata con l'esame del sedimento urinario previa acidificazione che dimostra la presenza dei caratteristici cristalli esagonali.

Nella nostra esperienza la presenza di cistinuria patologica è stata dimostrata con l'esecuzione sistematica del test di Brand in 39 (1.9\%) su 2086 pazienti consecutivi con calcolosi urinaria $(18,19)$. La diagnosi di cistinuria è stata confermata mediante dosaggio degli aminoacidi urinari con cromatografia a scambio ionico in 39 su 41 pazienti con test di Brand positivo.

L'età media dei pazienti alla diagnosi di calcolosi cistinica era $38.1 \pm 15.8$ anni, mentre l'età all' esordio clinico era di $21.8 \pm 12.4$. I calcoli renali erano recidivanti nell' $85 \%$ dei casi, mentre in 6 pazienti si trattava del primo episodio litiasico. Il rapporto maschi:femmine di 1:0.62.

Il numero medio di episodi litiasici di $18.5 \pm 35.8$ e l'intervallo medio tra il primo episodio e la recidiva di $4.1 \pm 4.3$ anni. Il tasso di recidiva a 5 anni dal primo episodio è stato dell' $83 \%$.

La funzione renale era alterata nel 18\% dei pazienti, uno dei quali era in trattamento dialitico.

In 18 casi si era reso necessario un trattamento chirurgico che è consistito nella nefrectomia in 4 casi. Tra 85 membri di 24 famiglie di pazienti con calcolosi cistinica, 24 presentavano valori anomali di cistinuria ma solo $5 \mathrm{di}$ loro avevano formato calcoli renali (21\%).

\section{Trattamento}

Il primo provvedimento per la profilassi della calcolosi cistinica deve essere rappresentato dall'incremento dell'assunzione di liquidi e dall'alcalinizzazione.

L'apporto idrico dovrebbe essere aumentato fino ad ottenere una diuresi di 3-4 litri/die ed il $\mathrm{pH}$ urinario incrementato $>7.5$ per incrementare la solubilità della cistina.

La restrizione dietetica della metionina ha in genere una ridotta compliance in quanto la dieta deve essere molto rigida ed è poco palatabile.

I farmaci tiolici, che aumentano la solubilità della cistina, rappresentano un trattamento di seconda linea nei pazienti non responsivi.

In particolare l'alfa-mercapto-propionil-glicina (alfaMPG) sembra avere una pari efficacia della D-penicillamina nel ridurre i livelli urinari di cistina con ridotti effetti collaterali (20-21).

Alcuni Autori (22) hanno descritto l'uso dell'antipertensivo captopril nel trattamento dei pazienti con cistinuria, sfruttando la formazione di complessi disulfidrilici tiolici che si formano in modo simile a quanto avviene con l'impiego di D-penicillamina e alfa-MPG.

L'impiego dei composti tiolici può consentire di ottenere la dissoluzione parziale o completa dei calcoli di cistina. Nella nostra esperienza il trattamento di 22 pazienti con $1-1.5 \mathrm{~g} /$ die di alfa-MPG ha comportato una riduzione del tasso di recidiva da 0.93 a 0.46 calcoli/paziente/anno (18). Solo 6 pazienti hanno manifestato effetti collaterali di gravità tale da richiedere la sospensione del trattamento (proteinuria, nefrosi, febbre e brividi, alterazioni ematologiche).

La litotrissia extracorporea (SWL) ha un'efficacia limitata nella frammentazione dei calcoli cistinici a causa dell'uniformità della sua struttura cristallina.

Nei casi di calcolosi cistinica resistenti al trattamento medico, la litotrissia endoscopica con ultrasuoni o laser rappresenta il trattamento di scelta, sebbene buoni risultati siano stati ottenuti con il trattamento chemo-litolitico attraverso l'infusione di soluzioni alcalinizzanti attraverso cateteri ureterali o nefrostomici (23-25).

In un recente studio abbiamo valutato l'impatto dell'introduzione delle nuove modalità di trattamento mini- 
invasivo sulla storia clinica dei pazienti con cistinuria (26).

Nel periodo 1978-89 (pre-SWL) in un gruppo di 31 pazienti erano stati sottoposti a trattamento chirurgico aperto 16 pazienti per un totale di 29 interventi $(0.93$ per paziente) di cui 4 nefrectomie; nel periodo 1990-2004 (post-SWL) in un gruppo di 17 pazienti solo 8 erano stati sottoposti a trattamento chirurgico aperto, ma altri 7 erano stati sottoposti a nefrolitotomia percutanea $(0.47+$ 0.41 per paziente). Nel gruppo 1990-2004 erano inoltre state eseguite 37 SWL (2.17 per paziente) mentre prima del 1989 erano state eseguite solo 4 SWL.

$\mathrm{E}$ interessante osservare come le caratteristiche cliniche dei pazienti non siano dissimili nei due periodi e come in particolare esista tuttora un importante ritardo nella diagnosi della malattia rispetto al suo esordio. An- che il tasso di recidivanza non si è ridotto in modo significativo, sebbene l'adozione di tecniche endourologiche abbia ridotto la morbilità del trattamento chirurgico con conseguente riduzione dei casi complicati con insufficienza renale (19 vs $6 \%$ ).

\author{
Indirizzo degli Autori: \\ Alberto Trinchieri, MD \\ UO Urologia \\ Ospedale A. Manzoni \\ Via dell'Eremo, 3/9 \\ Lecco, Italy \\ a.trinchieri@ospedale.lecco.it
}

\section{Bibliografia}

1. Calonge M, Gasparini J, Chillaron J, et al. Cystinuria caused by mutations in rBAT, a gene involved in the transport of cystine. Nat Genet 1994; 6: 420.

2. Rousad F, Rousad A, Nunes V, Barcel P, Palacin M. Progrès dans le génétique de la cystinuria. Ann Urol 1995; 29: 346.

3. Pras E, Arber N, Aksentijevich J, et al. Localization of a gene causing cystinuria to chromosome 2p. Nat Genet 1994; 6: 415.

4. Ito H, Egoshi K, Mizoguchi K, Akakura K. Advances in genetic aspects of Cystinuria. Mol Urol 2000; 4: 403.

5. Dello Strologo L, Pras E, Pontesilli C, et al. Comparision between SLC3A1 and SLC7A9 cystinuria patients and carriers: a need for a new classification. J Am Soc Nephrol 2002; 13: 2547.

6. Harnevik L, Fjellstedt E, Molbaek A, Denneberg T, Soderkvist P. Mutation analysis of SLC7A9 in cystinuria patients in Sweden. Genet Test 2003; 7: 13.

7. Rosemberg LE, Duant JL, Albrecht I. Genetic heterogeneity in cystinuria: evidence for allelism. Trans Ass Amer Physicians 1966; 79: 284.

8. Ito H, Murakami M, Miyauchi T, Mori I, Yamaguchi K, Usui T, Shimazaki J. The incidence of cystinuria in Japan. J Urol 1983; 129: 1012.
9. Bostrom H, Tottie K. Cystinuria in Sweden. II. The incidence of homozygous cystinuria in Swedish school children. Acta Paediatr Scand 1959; 48: 345.

10. Crawhall JC, Saunders EP, Thompson CJ. Heterozygotes for cystinuria. Ann Hum Genet 1966; 29: 257.

11. Lewis HB. The occurrence of cystinuria in healthy young men and women. Ann Intern Med 1932; 6: 183.

12. Turner B, Brown DA. Aminoacid excretion in infancy and early childhood: a survey of 100,000 infants. Med J Aust 1970; 1: 11.

13. Feliubadalo L, Arbones ML, Manas S, et al. Slc7a9-deficient mice develop cystinuria non-I and cystine urolithiasis. Hum Mol Genet 2003; 12: 2097.

14. Caldwell RJ, Townsend JI, Smith MJV. Genetics of cystinuria in an inbred population. J Urol 1978; 119: 531.

15. Malek RS, Kelalis PP. Pediatric nephrolithiasis. J Urol 1975; 113: 545.

16. Bhatta KM,PrienELJr,DretlerSP,etal.Cystinecalculi-rough and smooth:anewclinicaldistinction.JUrol 1989;142:937-9. 17.

17. Kim SC, Burns EK, Lingeman JE, Paterson RF, McAteer JA, Williams JCJr. Cystine calculi: correlation of CT-visible structure, CT number, and stone morphology with fragmentation by shock wave lithotripsy. Urol 
Res 2007; 35: 319-24.

18. Trinchieri A, Luongo P, Rovera F, et al. Pharmacological management of cystine nephrolithiasis: 10 years experience In Urology 1992. Bologna: Monduzzi Editore, 1992; 63.

19. Trinchieri A, Dormia G, Montanari E, Zanetti G. Cystinuria: definition, epidemiology and clinical aspects. Archivio Italiano di Urologia e Andrologia 2004; 76:129-34.

20. Crawhall JC, Scowen EF, Watts RWE. Effect of penicillamine on cystinuria. Br Med J 1963; 1: 588.

21. Pak CYC, Fuller C, Sakhaee K, Zerwekh JE, Adams BV. Management of cystine nephrolithiasis with alpha-mercapto-propionylglycine. J Urol 1986; 136: 1003.

22. Sloand JA, Izzo JL Jr. Captopril reduces urinary cystine excretion in cystinuria. Arch Intern Med 1987; 147: 1409.

23. Leroy AJ, Segura JW, Williams HJJr, Patterson DE. Percutaneous renal calculus removal in an extracorporeal shock wave lithotripsy practice. J Urol 1987; 138: 703.

24. Schmeller NT, Kersting H, Schuller J, Chaussy C, Schmiedt E. Combination of chemolysis and shock wave lithotripsy in the treatment of cystine renal calculi. J Urol 1984; 131: 434.

25. Dormia E, Dormia G, Malagola G, Minervini S. Experience with instrumental chemolysis for urolithiasis. J Urol 2003; 170: 1105.

26. Trinchieri A, Montanari E, Zanetti G, Lizzano R. The impact of new technology in the treatment of cystine stones. Urol Res 2005; 35: 129-32. 